Als pem axatomischex Institet ze Erlanglix.

\title{
BEITRÄGE ZUR ENTWICKELUNGSGESCHICHTE
}

\author{
DER
}

\section{CORNEA DER SÄUGER.}

VON

MORIZ WOLFRUM.

Mit 3 Figuren im Text und 6 Figuren auf Tafel $r$. 

Die Entwickelung der einzelnen Teile des Auges ist in der neueren Zeit vielfach Gegenstand der genaueren Untersuchung gewesen. So hat Rabli) vor Kurzem eine Reihe ausführlicher Arbeiten über die Bildung der Linse veröffentlicht, in der die Resultate von umfangreichen Untersuchungen über die Linse der Vertebraten niedergelegt sind. Sie haben ihm die Möglichkeit geboten, Ergebnisse von weittragender biologischer Bedeutung zu gewinnen. Von Felix Terrien ${ }^{2}$ ist eine Arbeit über die Entwickelung des Ciliarkörpers, von Cirincione ${ }^{3}$ ) über die der Linsenkapsel erschienen und so noch mehrere Publikationen. Auffällig ist dabei, dass die Entwickelung der ('ornea noch nicht eingehender gewürdigt worden ist, obwohl doch gerade sie als fertiges Gebilde von jeher ein Lieblingsobjokt der Histologen und Pathologen war. In der Literatur sind vor allem aus früherer. Zeit eine Reihe von Angaben vorhanden, die aber in manchen wichtigen Punkten sich widersprechende Anschauungen enthalten. Es schien deshalb einen Erfolg zu rersprechen, die Entwickelung der Cornea zu untersuchen. Auf Anregung von Herrn Dr. A. Spuler habe ich es daher unternommen, die Ent-

1) C. Rabl, ¿̈ber den Bau und die Entwickelung der Linse; I. Teil: Zeitschr. für wiss. Zool., Bd. 63, 1898. II. Teil: Bd. 65, 1899. III. Teil : Bd. 67, 1900 .

2) F. Terrien. Recherches sur la structure de la rétine ciliaire et l'origine des fibres de la zonule de Zinn. Paris, Steinheil et Arch. d'Opht. Sept 1898.

5) G. Cirincione, Zur Entwickelung des Wirbeltierauges, Ïber die Entwickelung der capsula perilenticularis, Leipzig 1898. 
wickelung, speziell die Histiogenese, der Cornea bei Säugetieren zu untersuchen.

Bevor ich näher auf das Thema eingehe, möchte ich kurz einiges über die Entwickelung des Auges im allgemeinen vorausschicken. Bekanntlich geht gleichzeitig mit der Einbuchtung der primären Augenblase die Bildung der Linse als Einstülpung rom Ektoderm her vor sich. Sie schnürt sich schliesslich vollkommen vom äusseren Keimblatt ab und liegt dann unmittelbar unter der äusseren Haut als ein kugeliges Gebilde. Mit der Abschnürung sind die ektodermalen Bestandteile des Auges angelegt. Das mittlere Keimblatt, das schon vorher in den Bereich der Augenanlage getreten war, bildet dann die äusseren Umhüllungen der Gebilde. $\mathrm{Zu}$ den Abkömmlingen des Mesoderms gehört auch das Corneagerebe, abgesehen vom Epithel, das natürlich ektodermaler Abkunft ist. Seine erste Anlage ist zwischen Ektoderm und vorderer Linsenfläche zu suchen.

In der Literatur finden wir die ersten auf die Entstehung der Cornea bezüglichen Angaben bei Remak. ${ }^{1}$ ) Nach seiner Ansicht entsteht die Hornhaut in späterer Zeit, durch Verschmelzung des vorderen Endes des Sklera mit lem Hornblatt. Auch A. v. Kölliker ${ }^{2}$ ), der bereits in den 60er Jahren Untersuchungen über die Entwickelung des Auges gemacht hat, schreibt der Cornea als einem Abkömmling des Mesoderms eine

1) Remak, Lntersuchungen über die Entwickelung der Wirbeltiere. Berlin 1855 .

2) A. Kölliker. Entwickelung der Linse, in Zeitschrift für wissensch Zool., Berlin 185.5.

A. Kölliker, Mikroskop. Anatomie, Bd. II., Leipzig 1854.

A. Kölliker. Entwicklungsgeschichte des Menschen und der höheren Tiere, Leipzig 1861.

A. Kölliker, Zur Entwickelung des Auges und Geruchsorgans menschl. Embryonen in: Festschrift zur Feier des 50jährigen Jubiläums der Universität Zürich, 1883. 
spätere Entstehung zu. Dagegen macht Babuchinl) die Angabe, dass er schon am 3. Tage bei Hühnchen die erste Anlage der Cornea in Form von runden Zellen gefunden habe.

Kessler ${ }^{2}$ ) hat in einer Arbeit vom Jahre 1871 sehr ausführlich über die Entstehung der Cornea berichtet. Er folgt dabei der Ansicht von Hensen, der die Anlage der Cornea als ein dem Glaskörper ähnliches Gebilde darstellt,. das dann in der Bildung der Cornea aufgehe. Kessler selbst macht in seiner Arbeit über die Entstehung des Auges bei Hühnchen und Tritonen (p. 18, Zeile 3 ff.) folgende zusammenfassende Angabe: An den Augenteil des Hornblattes bildet sich eine anscheinend strukturlose Schicht, die Anlage der Grundsubstanz der Cornea propria, an, - an diese das hintere Epithel; in die vorgebildete Grundsubstanz wandern von den Kopfplatten aus Zellen ein, welche zu den sternförmigen Hornhautkörperchen sich transformieren; nur eine schmale vordere und hintere Zone bleiben zellcnlos, sie stellen die rordere und hintere Grenzschicht dar."

Ton $A r^{\prime}$ old ${ }^{3}$ ) und Lieberkühn ${ }^{4}$ ) werden diese Angaben Kesslers einer eingehenden Kritik unterzogen. Sie kommen beide zu der Ansicht, dass in dem Gewebe, das die Anlage für die spätere Hornhaut bildet, jederzeit Zellen rorhanden sind. Arnold schreibt (l. c. p. 50, Zeile 12 ff.): "Dieselben sind

1) Babuchin, Beiträge zur Entwicklungsgeschichte der Retina, in Würzburger naturw. Zeitschr. Bd. IV. und V. 1863 und 1864.

2) Kessler, Cntersuchungen über die Entwicklung des Auges. Diss., Dorpat 1871.

Kessler, Zur Entwickelung des Anges der Wirbeltiere. Leipzig 1877.

3) Arnold, Beiträge zur Entwicklungsgeschichte des Auges. Heidelberg 1874 .

4) Lieberkühn, Über das Auge des Wirbeltierembryo. Schriften der Gesellsch. z. Beförd. der ges. Naturwiss. Marburg 1872.

Lieberkühn, Beiträge zur Anatomie des embryonalen Auges. Sitzungsber. der Ges. zur Beförd. der ges. Naturwiss. Marburg 1879. 
allerdings in den früheren Perioden spärlicher und in den schmalen vor der Linse gelegenen Gewebstreifen, namentlich an Durchschnitten, nicht immer leicht nachweisbar. Sie fehlen aber niemals und zeigen im wesentlichen dasselbe Verhalten, wie die Kerne des Gewebes der Kopfplatten." Und (p. 50, Zeile 26 ff.): „Auch bezüglich des Descemetschen Epithels kann ich Kessler nicht beipflichten: Meinen Beobachtungen zufolge entsteht es in loco gleichzeitig mit der sich vollziehenden Scheidung des ror der Linse gelegenen Fortsatzes des mittleren Keimblattes in Connea einerseits und membrana capsulo pupillaris andererseits.« Trotz dieser Einwände blieb Kessler in seiner Arbeit vom Jahre 1877 auf seinen früher gemachten Angaben bestehen.

Ton da ab findet sich in der Literatur eine grosse Lücke. Über viele Jahre hin sind mit Ausnahme der Arbeit Köllikers in der Züricher Festschrift keinerlei Angaben über die Entstehung der Cornea zu finden. Aus dem Jahre 1890 erst ist eine Dissertation ron Schoebel ${ }^{1}$ ) zu erwähnen. Er schliesst sich im wesentlichen den Ausführungen A rnolds und Lieberkühns all.

In der nouesten Literatur sind einige sehr wertrolle Angaben in den Arbeiten Rabls$^{2}$ ) über die I.inse vorhanden, die als Ausgangspunkt für die Darstellung meiner Befunde dienen sollen.

In der Tierreihe tritt das mittlere Keimblatt zu verschiedenen Zeiten in den Bereich des Auges, um sich an seinem Aufbaue zu beteiligen. Doch wissen wir durch die Untersuchungen ron

1) Schoebel, Zur postembryonalen Entwickelung des Auges der Amphibien. Diss., Leipzig 1890.

2) C. Rabl, "̈̈ber den Bau und die Entwickelung der Linse. I. Teil: Zeitschr. f. wiss. Zool. Bd. 63, 1898. II. Teil : Bd. 65, 1899. III. 'T'eil: Bd. 67, 1900. 
Rabl (le. III. Teil p. 2) und Schoebel (lc. p. 9), dass bereits zwischen Ektoderm und primärer Augenblase eine Mesodermschichte vorhanden ist, eine Erscheinung, die von diesen Autoren für die Vertebraten als allgemein gültig nachgewiesen ist. Hat sich nun die Linse vom Ektoderm abgeschnürt, so setzt kurz darauf die Bildung der Cornea ein. Rabl schreibt darüber (l. c. III. Teil p. 9) von einem Kaninchenembryo von $10 \mathrm{~mm}$ Nackensteisslänge: "Nachdem schon im vorigen Stadium die Mesodermzellen in Umkreis der Linse sich zwischen Ektoderm und Linsenepithel vorzuschieben begonnen hatten, haben sie sich jetzt zu einer kontinuierlichen Schicht rerbunden, welche die erste Anlage der tunica propria corneae vorstellt."

Zur Erläuterung füge ich bei, dass sich "im vorigen Stadium « die Linse eben vom Ektoderm gelöst hatte, und dass sie in diesem Kugelform angenommen hat.

An diese historische Darstellung schliesse ich zunächst einen kurzen Bericht über die angewandten Untersuchungsmethoden an. Zur Bearbeitung der vorliegenden Frage habe ich Embryonen von Schweinen, Schafen und Kaninchen benütat. Doch kamen auch die corneae junger und älterer geborener Tiere von Kaninchen, Meerschweinchen, Schafen, Kälbern und Hunden zur Verwendung. Die Fixirung des lebensfrischen Materials wurde teils in Herrmannscher Flüssigkeit, teils in etwas modificierter $Z$ enker scher Lösung, mit geringem Formolzusatz kurz vor dem Gebrauch, bei Körpertemperatur vorgenommen. Embryonen wurden ganz konserviert. Später wurden die bulbi unter Mitnahme von reichlichem Gewebe der Umgebung aus dem Kopfe ausgeschnitten und eingebettet. Von jüngeren resp. kleineren Tieren wurde das Auge frisch enucleiert, fixiert und dann die ganze Cornea mitsamt der Iris und einem Teil der Sklera rom Bulbus abgetrennt. Nach sorgfältigem, längerem Auswaschen erfolgte Härtung in steigendem Alkohol und durchwegs Einbettung in Paraffin. 
Die Schnittrichtung wurde an ganzen, embryonalen Augen in zwei Hauptrichtungen geführt. Die Schnitte verliefen entweder parallel zur optischen Achse, so dass man vollständige Durchschnitte von Augen bekam. Dabei waren die Augen teils so orientiert, dass die Lidspalte senkrecht getroffen war, teils so, dass Parallelschnitte zu ihr erfolgten. Oder die Corneae wurden tangential angeschnitten. So erhielt ich Flächenschnitte, die sich als besonders lehrreich für die Histiogenese des mesodermalen 'Teiles der Hornhaut erwiesen.

Die Corneae geborener Tiere wurden in den verschiedensten Richtungen geschnitten. Zwischen vollkommen senkrechten und Tangentialschnitten waren alle Richtungen vertreten. Die Schnittdicke schwankte zwischen $2 \mu$ und $7,5 \mu$. Die Objektträger wurden mit Spuren von Eiweiss bestrichen und mit destilliertem Wasser benetzt. Auf diese wurden dann die Schnitte in Serien übertragen und die so belegten Objektträger auf den Wärmeofen bei etwa $30^{\circ} \mathrm{C}$. getrocknet, wodurch eine sehr gute Ausbreitung und ein sehr festes Anhaften der Schnitte erreicht wurde.

Die Schnitte wurden zumeist nach der M. Heidenhainschen Eisen-Hämatoxylinmethode gefärbt, die sich auch bei dieser Arbeit vorzüglich bewährte. Die Behandlung in Hämatoxylin dauerte teils 24 teils 48 Stunden. Nachbehandlung wurde mit Rubin S, Mucicarmin und salzsaurer Orceïnlösung vorgenommen. Doch wurden letztere Färbemittel auch allein angewandt. Ausserdem habe ich die neue Cochenillestückfärbung, welche A. Spuler jüngst veröffentlicht hat, in Anwendung gebracht. Die Objekte wurden in wässeriger Cochenillelösung bei etwa $25^{\circ}$ Wärme zwei Tage gefärbt und nach Abspülung mit destillirtem Wasser in einer $0,3-0,5 \%$ Eisenalaunlösung zwei Tage gebeizt. Sodamn wurden die Stücke sorgfältig mindestens einen Tag mit destilliertem Wasser ausgewaschen und dann in steigenden Alkohol übertragen. Oder man wiederholte, um eine intensivere Färbung 
zu erzielen, den ganzen Prozess. Die Methode lieferte namentlich als Zellleib- und Bindesubstanzfärbung sowie kombiniert mit der Heidenhainschen Fisen-Hämatoxylinmethode als Schnittfärbung sehr scharfe Bilder für feinere Protoplasmastrukturen. Endlich sei auch erwähnt, dass Imprägnationen der Hornhaut mit Gold und Silber vorgenommen wurden.

\section{Form der Cornea.}

Ich beginne nun mit der Beschreibung der allgemeinen Formverhältnisse der Cornea, wie sie sich in situ und bei schwachen Vergrösserungen erkennen lassen.

Bei Schafsembryonen von $15 \mathrm{~mm}$ Scheitelsteisslänge bildet die Hornhaut eine kugelige Vorwölbung. Sie repräsentirt sich als ein Teil des den ganzen Bulbus einhüllenden mesodermalen Gewebes. Wie Schnitte zeigen, ist sie an den Rändern bedeutend dicker als in der Mitte, wo ihr die Linsenanlage anliegt. (Fig. 1.) Auch die Randdicke ist verschieden. Der nasale Hornhautrand hat einen grösseren Durchmesser als der temporale. Die Anordnung der Zellen parallel der Oberfläche ist schon deutlich ausgeprägt.

Fig. 1.

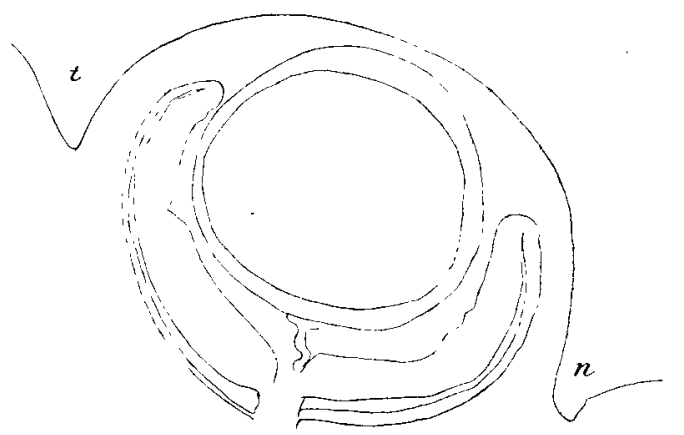

Bei Schafsembryonen von $18,5 \mathrm{~mm}$ Scheitelsteisslänge ist vor allem bemerkenswert, dass die ganze Cornea-Anlage bedeutend 
dicker erscheint. (Fig. 2.) Unter dem Epithel findet sich eine relativ breite zellfreie Zone. Das Hornhautgewebe lässt sich bei diesem Studium schon deutlich von dem Irisgewebe und der Linsengefässkapsel abgrenzen.

Fig. 2.

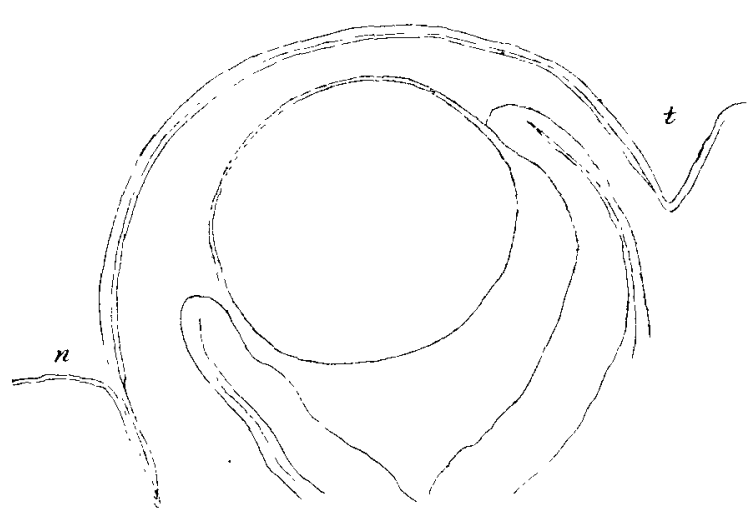

Schafsembryonen von $21 \mathrm{~mm}$ Scheitelsteisslänge haben eine im Verhältnis zu den Rändern viel dickere Hornhautmitte aufzuweisen. Diese hat also wiederum das grösste Wachstum erfahren. Am temporalen Rande ist die Bildung der Augenlider in der Form eines über den Hornhautrand wachsenden Hautwulstes angerleutet. Die subepitheliale zellfreie Zone ist nicht mehr scharf ausgeprägt.

Das ungefähr gleiche Verhalten treffen wir auch bei Schafsembryonen von $26 \mathrm{~mm}$ Scheitelsteisslänge, nur dass die Hornhautkuppe noch ctwas höher ist als im vorhergehenden Stadium. (Fig. 3.) Ausserdem ist das ganze Gebilde ron einem oberen und unteren Wulst, der Anlage der beiden Augenlider, umzogen. Ähnlich sind die Terhältnisse bei Schafsembrronen von $32 \mathrm{~mm}$ Scheitelsteisslänge, doch insofern auch rerschieden, als nur noch ein ganz kleiner Teil der Hormhaut infolge der sie schon zum 
grössten Teil bedeckenden Lider freiliegt. Bei Schweinsembryonen, von $38 \mathrm{~mm}$ Scheitelsteisslänge an, sind die Lider bereits geschlossen.

Fig. 3.

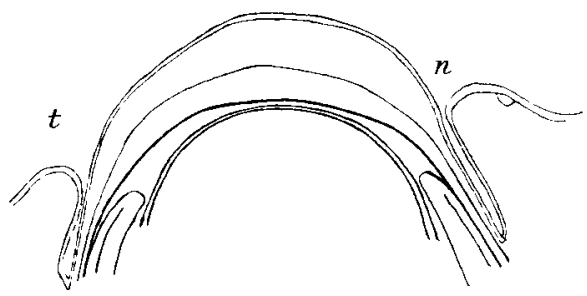

Bei schwachen Vergrösserungen stellt sich die Cornea auf Durchschnitten als ein Gebilde dar, das an seiner Innenseite nicht stärker gekrümmt als die Sklera, mit dieser ungefähr als vollständige Kugel die ektodermalen Bestandteile des Auges einhüllt. Wohl aber ist sie in der Mitte verdickt, sodass ihr äusserer Kontur stärker gewölbt erscheint. V'on einer eigentümlichen Krümmung der Cornea kam man wegen des Verlaufs der inneren Grenze nicht sprechen. Doch zeichnet sie sich dadurch aus, dass sie von dem Rande nach der Mitte kontinuierlich an Dicke zunimmt. Es haben diese Dickenverhälnisse mein besonderes Interesse erregt. Ich habe deshalb an einer Reihe von Schnitten Messungen vorgenommen, deren Resultate mit dem Ocularmikrometer gewonnen wurden. Doch sei herrorgehoben, dass sich geringe Fehler in Anbetracht der langen Behandlung, welcher dic Schnitte unterworfen wurden, nicht vermeiden liessen. Es ergaben sich jedoch nit geringen $\mathrm{Ab}$ weichungen gut übereinstimmende Resultate. Die Messungen wurden so gemacht, dass der Durchmesser der Cornealmitte und dann der des Rands festgestellt wurde. Nun ist freilich der Cornealrand bei solchen embryonalen Augen kein fixer Punkt. Es empfiehlt sich hier, den Umschlagsrand der retina als solchen anzunehmen. Derselbe behält so ziemlich während der ganzen 
Entwickelung des Auges, soweit es diese jungen Stadien betrifft, dieselbe Lage zur Cornea bei und entspricht auch aunähernd dem Cornealrande.

Schafsembryo von $26 \mathrm{~mm}$ Scheitelsteisslänge:

Mitte der Cornea $495 \mu$ $509 "$

$504 »$

$495 \%$

$477 "$

486 "

$477 "$

$477 \%$
Rand an der Umschlagsfalte

$108 \mu$

$108 »$

$108 》$

$108 \%$

$108 \%$

108

108 "

$108 \%$

Schafsembryo ron $32 \mathrm{~mm}$ Scheitelsteisslänge:

Mitte der Cornea

$486 \mu$
$468 "$
$486 "$
$495 \%$
$459 "$
$450 "$
$459 "$
$441 "$
$493 "$
$414 "$

Rand an der Umschlagsfalte der Retina

$198 \mu$

$216 *$

$207 *$

198 "

189 *

$189 »$

$198 \%$

$198 *$

$189 ”$

180 "

Die Zahlenreihen sind so geordnet, wie die Schnitte in den Serien aufeinander folgen. Sie sind aus der Mitte des Bulbus entnommen, so weit sich dies aus den Präparaten beurteilen liess.

Aus der ersten Zahlentabelle ergibt sich, dass das Dickenverhältnis rom Rande zur Mitte sich ungefähr wie 1:4,5 verhält. Die zweite Reihe zeigt, dass das Verhältnis rom Rand zur Nitte etwa 1:2 geworden ist. Vergleicht man nun die 
Zahlenwerte der ersten und zweiten Reihe, so ergibt sich, dass die Dicke der Mitte ungefähr die gleiche geblieben ist, ja, dass sie vielleicht etwas abgenommen hat.

Dagegen zeigt der Rand einen viel stärkeren Durchmesser in der zweiten Zahlenreihe. Es ist also diese Änderung in den Durchmesserverhältnissen fast allein auf Kosten des Randes zu setzen.

Ich lasse hier die Messungen von zwei Schweinsembryonen von 40 und $46 \mathrm{~mm}$ Scheitelsteisslänge folgen.

Schweinsembryo von $40 \mathrm{~mm}$ Scheitelsteisslänge:

$\begin{array}{ll}\text { Mitte } & \text { Rand } \\ 3 \check{1} 1 & 189 \\ 351 & 189 \\ 333 & 184,5 \\ 333 & 184,5 \\ 333 & 184,5 \\ 297 & 180 \\ 297 & 180 \\ 301,5 & 180 \\ 297 & 180 \\ 297 & 180 \\ 292,5 & 180 .\end{array}$

Schweinsembryo von $46 \mathrm{~mm}$ Scheitelsteisslänge;

$\begin{array}{cc}\text { Mitte } & \text { Rand } \\ 243 & 225 \\ 243 & 216 \\ 243 & 207 \\ 252 & 207 \\ 243 & 198 \\ 243 & 198 \\ 234 & 198 \\ 234 & 198 \\ 234 & 198 .\end{array}$


Es ergibt sich aus den beiden Tabellen, dass hier der Durchmesser der Mitte um ein bedeutendes abgenommen hat, während der Rand sich nahezu gleich geblieben ist. In der letzten Tabelle zeigen Rand und Mitte verhältnismässig geringe Durchmesserdifferenzen. Die Cornea ist in ihrer Form dem Zustande, den sie am ausgebildeten Tiere aufweist, schon ziemlich nahe gekommen. Welche Faktoren diesen Änderungen zu Grunde liegen, soll in einem späteren Artikel erörtert. werden. ${ }^{1}$ )

1) Kurz sei hier einiges über die Anlage der vorderen Kammer erwähnt. Dieselbe entsteht zuerst, wie sich auf Stadien von $32 \mathrm{~mm}$ Scheitelsteisslänge von Schafen nachweisen lässt, als ein ringförmiger schmaler Spalt. der von der Vorderfläche des Irisstromas und der Linse und von der Hinterfläche der Cornea begrenzt wird. Dentlich lässt sich dieselbe erst auf Schnitten von Schweinsembryonen von $46 \mathrm{~mm}$ Scheitelsteisslänge nachweisen. Dieser Spalt hängt in seiner Ausbildung offenbar mit der Entstehung des corpus ciliare zusammen. Dasselbe ist auch der Fall mit der besonderen Wölbung der Cornea. Mit der Anlage der Iris resp. des corpus ciliare erfährt die Linse einen Zug nach rückwärts, zu gleicher Zeit wird die Cornea durch die Entwickelung des corpus ciliare circulär eingeschnürt. Sie empfängt damit eine grössere Wölbung, sodass also ein Zurückweichen der Linse und ein Heraustreten der Cornea das Auftreten dieses spaltförmigen Raumes bedingen. Die Iris bleibt mit der Linse deswegen in festem Zusammenhang, weil sie durch Gefässe mit derselben verbunden ist. Fs verlaufen also diese Vorgänge mit einander und stehen in genetischer Beziehung. Die Vorderkammer ist zu dieser Zeit von einer eiweissreichen Flüssigkeit erfüllt. Der ringförmige Spalt nimmt von der Peripherie nach der Mitte hin zu. Es hebt sich hier der vordere Linsenpol am spätesten von der Hinterfläche der Cornea ab.

Auf Durchschnitten von Schweinsembryonen von $46 \mathrm{~mm}$ Scheitelsteisslänge findet man an der Innenseite der Cornea bereits eine kontinuirliche Schicht von Zellen angelegt. Dieselbe ist von der Cornea durch einen feinen, stark mit Rubin $\mathrm{S}$ sich tingierenden Saum abgegrenzt. An der freien Seite der Zellschicht, nach innen von der vorderen Fläche der Linse und des Irisstromas begrenzt, liegt eine geronnene feinkörnige Masse von rötlich brauner Tinktion nach Eisenalaunhämatoxylinbehandlung mit Rubin S. Wo dieselbe den Gewebsschichten anliegt, zeigt dieselbe vacuoläre Bildungen von ziemlich gleichmässiger Grösse. Wir haben in dem von dieser Masse erfüllten Raum 
Nach dieser Darstellung der gröberen Gestaltsrerhältnisse wende ich mich der eigentlichen Histiogenese der Cornea zu. Fs wurde dabei die Einteilung so getroffen, dass die einzelnen Gewebsbestandteile gesondert behandelt wurden.

Bekanntlich liegt die Cornea an ihrer Vorderfläche zuerst frei und wird erst später von den Lidern bedeckt. An ihrer Hinterfläche findet man sie bei Schafsembryonen bis zu einer Grösse von $32 \mathrm{~mm}$ Scheitelsteisslänge vollkommen der Vorderfläche der Linse angelagert. Eine Trennung zwischen beiden ist nur insofern vorhanden, als sich zwischen ihnen eine dünnc Gewebslage der capsula vasculosa lentis befindet. Dieselbe nimmt gegen den Rand an Dicke zu. Auf die Vorderfläche der Linse schiebt sich vom Äquator her auf eine kleine Strecke der Umschlagsrand der pars caeca retinae herein. Mit einer schmalen Lage lockeren, gefässführenden Gewebes auf seiner Vorderseite füllt er deu Raum zwischen Cornea und Linse vollkommen aus. Von dem lockeren Gewebe aus kommunizieren Gefässe mit dem Gefässsrstem der capsula vasculosa lentis. Ausserdem flndet man etwas

die Anlage der Vorderkammer vor uns. Gewöhnlich ist sie allerdings durch Schrumpfungsprozesse vergrössert. Man findet dann Reste von der geronnenen Vasse sowohl am Descemetschen Epithel wie an der Iris hängend. Thre Grösse in idealem Zustande lässt sich jedoch an der Grösse des in ihr befindlichen Gerinnsels feststellen. Doch gelang es mir bei sehr sorgfältig fixierten und vor allem vorsichtig gehärteten Objekten Präparate zu erzielen. bei denen die Verhältnisse so liegen, wie sie oben geschildert wurden, Die Linse ist zu dieser Zeit mit ihrem vorderen Pol und dessen Umgehung noch fest der Hinterfläche der Cornea angelagert und haftet dort gewöhnlich bei Schrumpfungen. Es repräsentiert sich also die erste Anlage der Vorderkammer als ein schmaler ringförmiger Spalt, der da seinen grössten Durchmesser hat, wo der lrisrand an die Vorderfläche der Linse anstösst. Der vordere Pol der Linse löst sich erst später von der Hinterfäche der Cornea ab. Diese Verhältnisse sind auch bereits an Schafsembryonen von $32 \mathrm{~mm}$ Scheitelsteisslänge vorhanden. 
weiter vom Umschlagsrande nach aussen in diesem Gewebe auf temporaler und nasaler Seite mit grosser Konstanz ein ziemlich starkes Gefässlumen durchschnitten.

\section{Substantia propria corneae.}

Bei zwei Schafsembryonen von einer Scheitelsteisslänge von 15 und $22 \mathrm{~mm}$ liess sich bereits eine gewisse Anordnung der Bindegewebszellen feststellen. Bei dem jüngeren Stadium liegen sie in etwa 5-6 Schichten zwischen Ektoderm und Linse in schalenförmiger Anordnung über einander. Auf Flächenschnitten erscheinen die einzelnen Zellen als flache, breite und mit reichlichem Protoplasma versehene Gebilde. Gewöhnlich haben sie eine längliche Form, sodass der Breitendurchmesser etwa die Hälfte des Längsdurchmessers ausmacht. Die Kerne sind dementsprechend oval und zeigen mehrere Kernkörperchen. Zentralkörper sind vorhanden. Zumeist sind zwei zu sehen. Der Zellenleib ist in mehrere Zipfel ausgezogen, die in zahlreichen Verbindungen mit einander stehen und noch keine Fibrillenbildung erkennen lassen. Die ersten Anfänge ron Fibrillenbildung habe ich an Embryonen von $22 \mathrm{~mm}$ Scheitelsteisslänge wahmehmen können. Doch zeigen am besten diesbezügliche Terhältnisse Schafsembryonen ron $26 \mathrm{~mm}$ Scheitelsteisslänge. Hier kam man in der Corneaanlage bereits zwei Zonen unterscheiden. In der Mitte des Organs repräsentieren sie sich als eine oberflächliche etwa ${ }^{3} / 5$ einnehmende lockere und eine tiefere die übrigen ${ }^{2} \%_{s}$ für sich bis auf einen kleinen Saum vor der Immenfläche in Anspruch nehmende imnere Schichte mit bereits rorgeschrittener Fibrillenbildung. Auf Durchschnitten lassen sich die beiden Teile leicht an der stärkeren Rotfärbung der Innenzone mit Rubin S gefärbter Präparate unterscheiden. Es sei von vorneherein erwähnt, dass diese tiefere Schichte sich als die unmittelbare Fortsetzung der Skleralanlage darstellt, während die 
äussere als der Cutisteil der Cornea aufzufassen ist und auch am Rande deutlich in die Konjunktiva übergeht. Beide Teile machen den Eindruck, als ob sie gegen die Mitte der Cornea nicht ihre vollkommene Dichte beibehielten, sondern sich etwas lockerten und dafür an Dicke gewännen und zwar ror allem die äussere Schicht. Sie lassen sich leicht an folgendem als differente Gebilde unterscheiden. Die Zellen zeigen in der tieferen Zone eine ganz regelmässige Anordnung, sie liegen flach. wie in der Skleralanlage, über einander geschichtet und zeigen kaum irgendwo ein Abweichen ron diesem Verhalten. In dem oberflächlich gelegenen Teil liegen sie zwar auch grösstenteils parallel zur Oberfläche. Man trifft sie indess hie und da unter den verschiedensten Winkeln zum Epithel liegend. Manche, eine Erscheinung, die gerade in der Mitte am häufigsten vorkommt, haben sich vollkommen senkrecht zur Oberfläche gestellt. Vergleicht man nun die Zellen der Cornea in verschiedenen Schichten, so findet man, je weiter man von innen nach aussen rückt, eine desto grössere Lnregelmässigkeit in der Anordnung und Gestaltung der einzelnen Gewebselemente. Der Grund hiefür dürfte in folgendem zu suchen sein:

Die Sklera bildet die Begrenzung einer Kugel, die sich mitten im Gewebe befindet und deshalb auch den im Inneren des Bulbus und im umgebenden Gewebe herrschenden Druckverhältnissen ausgesetzt ist. Da diese Kugel das Bestreben hat, sich auszudchnen, und in der That eine kontinuierliche Tergrösserung ihres Innenraumes erfolgt, so wird die Sklera im Verlaufe ihres Wachstums unter diesen Einflüssen eine entsprechende Anorduung ihrer Gewebselemente zeigen. Sie werden der Ausdehnung der Kugel folgend ihre Längsachse parallel zur Kugeloberfläche richten und um so mehr auch in flachen Schichten angeordnet werden, weil auf ihnen ringsum noch der Gewebsdruck lastet. Bei der Hornhaut indes liegen die V'erhältnisse etwas anders. Sie ist auch dem Innendruck des Auges ausge- 
setzt, aber diesem steht nur der Druck in der Amnionhöhle gegenüber, und dieser ist sicherlich erheblich geringer, als der Gewebsturgor des wachsenden Embryos. Infolge dessen zeigt nur die innere Schichte die regelmässige kontinuierliche Schichtung und die weiter fortgeschrittene Differenzirung, weil hier der intraoculare Druck auf die Zellen richtend wirkt.

Nach aussen dagegen steht dem Gewebe bei seinem Wachstum nur ein geringer Widerstand entgegen. Es entwickelt sich daher locker, weniger regelmässig und etwas roluminöser. Da die Ränder in ihrem Wachstum teilweise noch unter denselben Bedingungen wie die Sklera stehen, so wird dies hier weniger zum Ausdruck kommen als gerade in der Mitte. Die Messungen, welche zu Anfang der Arbeit festgelegt wurden, sprechen dafür und finden damit auch ihre Erklärung. Zieht man Schafsembrỵonen von $32 \mathrm{~mm}$ Scheitelsteisslänge zum Vergleiche heran, so ergibt sich keine wesentliche Veränderung dieses Verhaltens. Demn auch hier lassen sich die beiden Schichten unterscheiden, beide werden nach den Rändern schmäler, die äussere um ein gut Teil mehr als die imnere, und gehen dam in die Sklera über. Die äussere Schicht zeigt im Bereich der Lidspalte noch den lockeren Aufbau, aber soweit sie von den Lidanlagen überlagert ist, hat sie einen regelmässigen Bau gewonnen und erscheint unter den heraufrückenden Lidern etwas dünner geworden.

Ganz ander's ist das Bild der Cornea anf Schnitten ron Schweinsembryonen von 40 und $46 \mathrm{~mm}$ Scheitelsteisslänge. Die Lider sind hier bereits verwachen. Die Struktur des Gewebes zeigt auch in den oberflächlichen schichten einen durchaus regelmässigen (harakter. Ausserdem ist tie aussere Schicht dichter geworden. Doch ist noch ein scharfer Unterschied zwischen äusserer und innerer Zone vorhanden, von denen sich letztere wesentlich durch ihre stärkere Fibrillenbildung auszeichnet. Die Dicke der ersteren ist dabei bedeutend gleich- 
mässiger geworden. Das gleiche Verhalten ergaben auch Schnitte von Schafsembryonen von $62 \mathrm{~mm}$ Scheitelsteisslänge. An neugeborenen Meerschweinchen und nicht ganz ausgetragenen Hunden war wohl die Schichtenunterscheidung noch möglich, aber nicht mehr so prägnant.

Es ergibt sich aus obigem, dass vor allem die lockere äussere Schicht durch den mechanischen Druck der heraufwachsenden und sich schliessenden Lider, abgesehen ron den in ihr selbst waltenden Wachstumsvorgängen, nicht nur eine relative, sondern auch eine absolute Abnahme ihres Dickendurchmessers erfährt, dass aber dieser Faktor zugleich wesentlich zu ihrem regelmässigen Baue beiträgt. Damit finden auch die Resultate der auf der 12. und 13. Seite angeführten Messungen ihre Erklärung. Die Cornea im ganzen wird eben mit dem Heraufwachsen der Lider, während sie vorher nur auf ihrer Innenseite dem intraoculären Drucke ausgesetzt war, den gleichen Wachstumsbedingungen unterworfen wie die Sklera. Sie muss sich wie eine zwischen zwci Wänden befindliche Lamelle entwickeln und daraus resultiert ihre Gewebsanordnung.

Ich wonde mich nun der Histiogenese des bindegewebigen Teiles der Cornea zu. Bei der Durchmusterung meiner Schnittserien finde ich, dass die Cornea in jungen Stadien ein recht geeignetes Objekt zum Studium der Fibrillenbildung abgibt. Zunächst sei die Entstehung der Fibrillen selbst besprochen.

Als Ausgangspunkt wälle ich eine Zelle, welche direkt unter dem Epithel liegend, sich in einem Flächenschnitte ron $2 \mu$ ron einem Schafsembryo von $26 \mathrm{~mm}$ Scheitelsteisslänge fand. Siehe Tafel, Fig. 1. Ihr Kern hat eine clliptische Form und zeigt ziemlich glcichmässig über die Fläche verteilte, zarte Chromatinkörner, nicht serial angeordnet. Lusserdem sind ihm grössere Nukleolen eingelagert. Er ist von roichlichem Protoplasma umgeben, dessen grösste Anhäufung der Längsachse des Kernes entspricht. Das dargestellte Verhalten des Protoplasmas, wo die 
Zelle in einer Richtung gestreckt erscheint, war in diesen Stadien weitaus das häufigste. Doch trifft man auch Zellen mit Kernen von mehr runden Formen. Das Protoplasma ist dann in mehreren Richtungen zu Zipfeln ausgezogen. Man findet Zellen mit drei oder vier und noch mehr Protoplasmafortsätzen. Diese Ausläufer stehen, soweit sie sich verfolgen lassen, fast alle mit den nächstliegenden Zellen in Terbindung.

Die dargestellte Zelle zeigt in der Bildebene nur ein Zentralkorn, das an der Seite des Kernes gelegen ist. Es ist umgeben von einem kleinen hellen Hofe, in dessen Umgebung das Protoplasma deutlich fein granuliert ist Die Zentralkörper sind sonst fast in jeder Zelle als zwei scharf tingierte, schwarze längliche Körperchen in der Nähe des Kernes oder auch in weiter Entfernung von ihm in einem der Hauptprotoplasmaauslïufer zu finden. In ihrer Umgebung zeigt das Protoplasma durchwegs granulären Charakter. Derselbe nimmt aber, wie ans der Abbildung ersichtlich ist, mit der Entfernung von den Zentralkörpern an Regelmässigkeit ab, die Körnchen werden allmählich serial geordnet und gehen in fädige Strukturen über. Diese nehmen gegen die Zellgrenzen hin mehr an Schärfe zu und gehen schliesslich in Fibrillen über, die in wechselnder Menge von den Zellen abgehen. Zwischen den fibrillären Strukturen findet man, wenn auch selten, vacuoläre Bezirke, die vor allem da, wo die Fibrillenzüge aus der Zelle heraustreten, manchmal zwischen diese eingelagert sind. Die fädige Struktur ist in dem grössten Teile des Protoplasmas rorhanden und geht bis dicht an den Kern heran. Ein Verschmelzen mit der Kernmembran konnte ich nicht finden. Einen derartigen bis an die Kernmembran herangehenden Zug findet man auch in der Abbildung bei $x$. Es ist aus diesen Befunden anzunehmen, dass die Fibrillen ihre Vorbildung innerhalb des Protoplasmas erfahren und mit zunehmender Ausbildung an die Längsseitenränder der Zellen wandern, wo sie allmählich zuerst in grösster Ent- 
fernung vom Kerne, aus dem Protoplasma abgespalten werden. Eine Bestätigung dafür erhält man auch an durchschnittenen Zellen. Ich habe damit nur bereits Gefundenes bestätigt. Es sind diese Vorgänge eingehend von A. Spuler ${ }^{1}$ ) dargelegt und

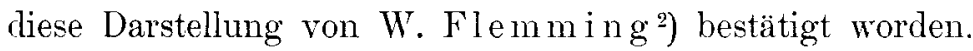

Auf einen besonderen Punkt möchte ich ebenfalls aufmerk. sam machen, da er gerade bei der Cornea eine ziemliche Rolle spielt. Die flachen Bindegewebszellen spalten, gemäss ihrer Lage, die Fasem weitaus zum grössten Teil nur in Ebenen ab, die parallel zur Oberfläche liegen. Dabei ist der Vorgang keineswegs so zu denken, dass jede einzelne Zelle für sich Fibrillen liefert. Die Zellen sind ja in weiter Ausdehnung mit einander in protoplasmatischer Verbindung, so dass von einer ganzen Reihe von Zellen zugleich die Abspaltung ron längeren Fibrillen erfolgt. Ich bin überzeugt, würde man die Hornhaut parallel ihrer lamellösen Anordnung schneiden können, man fände schon zu dieser Zeit Fasern, die eine noch viel bedeutendere Länge aufweisen. So sieht man sie nur in der Länge ron 3 oder 4 Zellen angelegt. Doch gelang es, an günstig gelagerten Objekten bei Schafsembryonen von $62 \mathrm{~mm}$ Scheitelsteisslänge sehr lange Fasern durch das Gesichtsfeld zu verfolgen.

Bei Beginn dieses Kapitels wurde von Zellen gesprochen, die in verschiedenen Winkeln zur Oberfläche der Hornhaut liegen. Da auch von ihnen Fibrillenbildung ausgeht, so müssen wir ihnen die Funktion zuschreiben, auf diese Weise einen Zusammenhang und eine gegenseitige Verankerung der gebildeten Fibrillenlamellen zu schaffen. Doch ist nicht unerwähnt zu lassen, dass von ganz parallel zur Oberfläche liegenden Zellen

1) A. Spuler. Beiträge zur Histiologie und Histiogenese der Bindeund Stützsubstanz. Anat. Hefte etc. Wiesbaden 1896.

2) W. Flemming, Über die Entwickelung der collagenen Bindegewebsfibrillen bei Amphibien und Säugetieren. Archiv für Anatomie und Physiologie 1897. 
schief abgehende Fortsätze mit Abspaltung von Fibrillen ausgehen, die in die nächst liegenden Schichten übergreifen, so dass auch so eine imnigere Vereinigung der Lamellen erfolgt.

Bei älteren Stadien ron Schafsembryonen ron 62 sowie $90 \mathrm{~mm}$ Scheitelsteisslänge und vor der Geburt stehenden jungen Hunden zeigte sich ein ungeheueres Fibrillengewirr angelegt, doch war die Anlage noch nicht eine derartig dichte und regelmässige, lass man von Lamellen hätte reden können. Die einzelnen Fibrillenbündelchen sind noch getrennt $z u$ beobachten. Dieselben zeigen ihre grösste Anhäufung an den Seiten der Protoplasmaverbindungen der Zcllen, die hier in ungemein grosser Zahl ron Zelle zu Zelle verliefen. Zum Teil waren die Fibrillen noch in Verbindung mit dem Protoplasma zu beobachten, es erfolgte also noch eine ausgerlehnte Neubildung derselben. Obwohl hier die Cornea ihrem fertigen Zustande sehr nahe steht, so kann man doch crst von dem vollkommen entwickelten Gebilde reden, wenn die Fibrillen zu Lamellen an einander gelegt sind, und ihre Bildung, die am fertigen Organ doch nur spärlich erfolgt, einen gewissen Abschluss erreicht hat.

Nan findet dann in der Hornhaut die gebildeten Fibrillenlamellen und zwischen ihmen liegend die Zellen mit ihren ungemein zahlreichen protoplasmatischen Terbindungen.

Auf der Tafel, Fig. 2, ist eine solche Zelle zur Darstellung gebracht. Es fallen da vor allem die zahlreichen Protoplasmaausläufer auf. An ihnen konnte fast durchwegs festgestellt werden, dass sie mit den Nachbarzellen in Verbindung stehen. Der längliche Kern zeigt grobe gleichmässige Granula. An seiner einen Seite sind sehr deutlich die beiden Zentralkörper zu sehen und oben im Bilk bereits die der nächsten Zelle. Ballowitz ${ }^{1}$ ) hat darüber bereits eingehende Cntersuchungen gemacht. Die

1) Ballowitz, Kernmetamorphosen der Hornhaut während ihres Wachstums und im Alter. Grä fe's Archiv, Bd. 50, Abt. II. 1899. 
gelbe Tönung um die Zelle der Fig. 2 soll schematisch den Fibrillenverlauf wiedergeben.

Da die Cornea, wie alle Gewebe, einer gewissen Abnützung unterworfen ist, - wenn man auch annehmen darf, dass wegen der konstanten Verhältnisse diese geringer ist als bei den meisten anderen Organen --, so muss doch eine beständige Regeneration der Hornhautfibrillen in geringem Umfange stattfinden. Dafür müssen die Hornhautzellen mit ihren protoplasmatischen Verbindungen in Anspruch genommen werden, eine Funktion, die ihnen natürlich nicht nur während des embryonalen und postembryonalen Wachstums, sondern auch am fertigen Gebilde und vor allem bei pathologischen Verhältnissen zukommt.

In der fertigen Hornhaut findet man, wenn auch nur selten, Fasern, welche zu dem Typus der sog. elastischen zu gehören scheinen. Un solche aufzufinden, auch den Schleimgehalt des embryonalen Gewebes beurteilen zu können, wurden Nachfärbungen nach vorhergehender Behandlung mit Eisenhaematoxylin ausser mit Rubin $S$ auch mit Orceïn und Mucicarmin vorgenommen. Wurde Orceïn in salzsauerer alkoholischer Lösung als alleinige Färbung oder als Nachfärbung angewandt, so war damit trotz prolongierter Einwirkung nur eine äusserst schwache Tinktion der Fibrillen zu erzielen. Die Corneafibrillen zeigen also in jungen Stadien nirgends die Ejgentümlichkeiten elastischer Fasern; doch auch an den Präparaten fertiger Corneae gelang es mir nicht derartige velastische« Fasern zu finden. Die Färbung musste als eine gelungene bezeichnet werden, da in den Lidern und am Skeralrand mitgetroffene Gefässe stets eine tiefbraunrote Tinktion der elastischen Elemente aufwiesen. Eine sehr scharfe Färbung vieler Fibrillen war mit Mucicarmin zu erzielen, indem der auf ihnen niedergeschlagene Schleim feinkörnig tingiert wurde. Im übrigen zeigten solche Präparate bei schwacher und mittlerer Vergrösserung eine diffus rosa Färbung. Entsprechende Resultate wurden mit einfacher Hämatoxylinbehand- 
lung nach vorhergehender Osmierung erzielt. Bekanntlich sind die Erscheinungen auf eine Mitfärbung der mucösen Kittsubstanz und des Mucins der Gewebsflüssigkeit, das bei Embryonen in grösserer Menge dort vorhanden ist, zurückzuführen.

Ich wende mich nun den Befunden zu, die, abgesehen von der Fibrillenentwickelung, in dem bindegewebigen 'Teile der Cornea sonst zu konstatieren waren. In den oberflächlichen Schichten findet man vor allem bei Serien von Embryonen von 26-32 mm Scheitelsteisslänge Lücken, die als einfache Löcher im Schnitte sich darstellen, aber nicht als artifizielle Produkte gedeutet werden dürfen, weil sie sich in Serien durch Schnittreihen verfolgen lassen und nirgends in der Umgebung Zeichen einer lokalen Schrumpfung sich finden. Hie und da findet man diese Hohlräume umgeben von feinen Fasern, manchmal liegt auch eine Zelle dem Lumen an. Protoplasma und Kern zeigen dann eine der Umgrenzung des Hohlraums entsprechende Biegung. Am zahlreichsten und grössten sind diese Gebilde bei Schafen von $26 \mathrm{~mm}$ Scheitelsteisslänge anzutreffen. Gegen die Mitte und 'Tiefe der Homhaut werden sie beim Schafe seltener, wenigstens lassen sie sich nicht mehr so häufig als scharf charakterisierte Gebilde wahrnehmen. Dagegen scheint beim Schwein vor allen in Grössen von $40-46 \mathrm{~mm}$ Scheitelsteisslänge ihre Verteilung eine mehr gleichmässige zu sein. Bald liegen sie hier in der Nähe von Zellen, bald zwischen den Fibrillenschichten. Ein gesetzmässiger Verlauf scheint ihnen nicht zuzukommen. Man darf diese Befunde wohl mit Recht als ein Saftlückensystem der sich entwickelnden Cornea deuten. In wie weit dieses mit dem Saftlückensystem, das von vielen Autoren in der fertigen Hornhaut angenommen wird, gemeinsames hat, konnte ich deshalb nicht entscheiden, weil in Schnitten von späteren Stadien derartige Bilder nicht mehr zu sehen waren. Blutkörperchen oder sonstige Gebilde, welche auf das Vorhandensein von Blutleitern schliessen lassen konnten, 
wurden in der Säugetiercornea nicht gefunden. Es stimmen diese Befunde mit den Angaben J. v. Gerlach's') überein, der durch seine Injectionen nachgewiesen hat, dass die Gefässe mit einem Randschlingennetz, das sich noch teilweise am Rande in die Hornhaut hineinerstreckt, aufhören.

\section{Grenzschichten der Cornea.}

Der bindegewebige Teil der Comea grenzt aussen an das Epithel, imen wird er von dem Descemetschen Epithel abgeschlossen. Da hier besondere Modifikationen der Substantia propria corneae vorhanden sind, die man als Bowmannsche Membran und Descemetsche Lamelle bezcichnet, so bedürfen diese Grenzgebiete einer besonderen Beschreibung.

Schafsembryonen von $15 \mathrm{~mm}$ Scheitelsteisslänge lassen nichts auf diese Grenzlamellen bezügliches erkemen. Dagegen findet man bei solchen ron 18,5, 21 und $22 \mathrm{~mm}$ Scheitelsteisslänge unter dem äusseren Epithel einen roten Saum, der starke Rubin S-Reaktion zeigt. Ton ihm aus ziehen vereinzelte Fäden in das unterliegende Bindegewebe. Der Saum erscheint dem Epithel vollkommen angelagert und auch bei stärksten Vergrösserungen homogen. Bei Schafscmbryonen von 26 und $32 \mathrm{~mm}$ Scheitelsteisslänge sind schon besondere Verhältnisse entwickelt. Mit schwachen Vergrösserungen ist bei Färbung mit Rubins an der basalen Epithellage ein roter anscheinend homogener Saum zu sehen, der sich aber bei etwa 400 facher Vergrösserung in ein ungeheures Gewirr feinster Fibrillen auflësen lässt. Diese fibrilläre Zone wird nach inmen begrenzt von einer Lage von Bindegewebszellen, die als platte Gebilde unter ihr liegen. Hat man Schnitte, die rein senkrecht zur Oberfläche gefülırt sind, so grenzt sich der Fibrillensaum scharf

1) J. v. Gerlach, Handbuch der allgemeinen und speziellen Gewebelehre. Wien 1860. 
gegen die unterliegende Zellschichte $a b$, die Fibrillen hören plötzlich wie abgeschnitten auf. Es lassen sich nur selten Fasern von grösserer Länge in das darunter liegende Gewebe verfolgen. Sowie aber die Schnittrichtung unter einem gewissen Winkel zur Oberfläche geneigt ist, und derartige Schnitte bekommt man ohne weiteres bei Serien von Durchschnitten, so ist folgendes zu beobachten. Die Fibrillenbündel steigen als ziemlich starke Gebilde aus dem unterliegenden Bindegewebe auf, zersplittern sich allmählich in immer feinere Züge und gelangen so unter Bildung dieses subepithelialen Fibrillennetzes bis an die Epithelzellen. Oft kann man auch sehen, dass die Züge erst kurz ror dem Epithel pinselförmig auseinander strahlen. Aus der Thatsache, dass man solche Bilder nur aus Schrägschnitten gewimnt, ergibt sich, dass die Fibrillenbündel fast nie senkrecht emporsteigen, sondern nur unter einem gewissen Winkel zum Epithel in die subepitheliale Region gelangen.

Es wird sich nun um die Entscheidung der prinzipiell wichtigen Frage handeln, welche Verbindung diese Fibrillenschichte und damit das mesodermale Gerrebe mit dem Epithel, also dem ektodermalen Cornealteil, aufweist. Ein Zusammenhang muss von vorne herein postuliert werden, aber es lässt sich nur auf histiologisehem Wege entscheiden, welcher Art derselbe ist. Zur. Untersuchung dieser Frage dienten vor allem Schafsembryonen von 25 und $32 \mathrm{~mm}$ Scheitelsteisslänge, da hier die Terhältnisse für die Lösung dieser Aufgabe besonders günstig gelagert waren. An den jüngeren Embryonen liess sich bei Schrägschnitten konstatieren, wie auch die Fig. 3 der Tafel zeigt, dass in der That direkte Verbindungen der subepithelialen Fibrillenschichte mit der basalen Epithelschichte nicht in Abrede gestellt werden konnten. Die Fibrillen konnten vollkommeu scharf bis an das Protoplasma der Zellen, das hier ungemein spärlich ist, verfolgt werden. $O b$ es sich dabei wirklich um ein 
Hineindringen in das Protoplasma handelte, wage ich ohne weiteres nicht mit Bestimmtheit zu entscheiden. Nur soviel sich mit Sicherheit sehen liess, wurde in der Zeichnung wiedergegeben. Die nur spärlich von Protoplasma umgebenen Kerne zeigten da die grösste Anhäufung desselben, wo sich die Fibrillen mit den Zellen verbinden. An einer derselben sieht man auch einen ziemlich langen Protoplasmafortsatz in die Fibrillenschichte ziehen. Durch Heranziehung folgender Befunde lässt sich jedoch die Art des Zusammenhanges entscheiden. Es wurden in denselben Schnitten in ziemlicher Anzahl Bindegewebszellen gefunden, die, direkt unter dem Epithel liegend, deutlich in protoplasmatischer Verbindung mit den basalen Epithelzellen standen. Am zahlreichsten fanden sich solche Verbindungen an den Rändern, soweit sich über sie bereits die Lider hinweggesehoben hatten. Manchmal glückte es auch Stellen zu finden, in denen der protoplasmatische Zusanmenhang rorhanden, und bereits Fibrillenbildung eingetreten war. Durch derartige Befunde wird die Annahme sehr wahrscheinlich, dass die Fibrillen der subepithelialen Schicht in direkter Verbindung mit dem Protoplasma der basalen Epithelzellen stehen. Dass die Entstehung der Fibrillen rein auf Kosten der Bindegewebszellen zu setzen ist, dürfte ausser allem Zweifel stehen. Diese Verhältnisse komnten nur beim Schafe gefunden werden. Beim Schweine wurden zwar auch protoplasmatische Verbindungen gefunden, aber über die Verbindungen der Fibrillenzüge liess sich wegen der ungemeinen Feinheit der Gebilde nichts mit Sicherheit eruieren.

Aus dem eben Angeführten und aus der ganzen Darstellung der Fibrillenbildung geht hervor, dass die Entstehung des specifischen Hornhautgewebes in loco erfolgt. Es ist ohne Zweifel, dass im Beginne der Bildung der Hornhaut ein Eindringen vou noch nicht differenzierten Mesenchymelementen stattfindet, nicht aber erfolgt ein Einwachsen von Fasern, wie Schoebel in 
seiner Arbeit (l. c. p. 32) es darstellt. Zählungen der übereinander gelagerten Schichten und die Zuhilfenahme der Messungen ergeben vielmehr, dass die Bildung des bindegewebigen Teiles in konzentrischen Zonen erfolge, die von der Mitte nach dem Rande zu eine fortgeschrittenere Entwickelung zeigen.

Ich gehe nun zur Entwickelung der Descemetschen Nembran über. Dabei behandle ich die Membran und das Epithel mit einander, weil beide in ihrer Genese in inniger Be. ziehung stehen.

Ich fand dafür Schnitte von Schafsembryonen von 26 bis $29 \mathrm{~mm}$ Scheitelsteisslänge am geeignetsten. Dieselben lassen bei schwacher Vergrösserung folgendes erkemnen. Dic innerste Zellschichte der Cornea trägt im wesentlichen denselben Charakter, wie die anderen Hornhautschichten. An den Seitenränder'n ist zwischen Linse und Hornhaut die Anlage der Iris hercingeschoben, olme scheinbar ron letzterer eine Abgrenzung zu zeigen. Bei starker Vergrösserung macht man jedoch an den beiden inneren Zellschichten folgende merkwürdige Befunde: Nan findet im Protoplasma derselben mit Rubin S intensiv rotgefärbte Bestandteile. Auf Durchschnitten repräsentieren sich dieselben als Punkte, oder als längliche Gebille, die etwa die halbe Z/clldicke einnehmen. Oder sie zeigen sich zu Fasern ron mächtiger Dicke ausgewachsen, die in distaler Richtung rom Zellkerne sehr schnell an Dicke abnehmen, mit ihrer breitcn Basis dicht an ihn herangehen und ihm gleichsan aufsitzen. In der in der Nachbarschaft der Linse gelegenen Zellschichte sieht man diese Gebilrle, die wohl nichts weiter als besonders starke collagene Fasern sind, fast ausschlicosslich gegen die Cornea zu abgelagert. Siehe Fig. 4 auf der Tafel. An der cornealwärts folgenden Zellschichte ist ihre Fntstehung. nur auf der Linsenseite zu konstatieren. Zwei Zellschichten sondern also auf den einander zugewandten Flächen Produkte ab, die sich schliesslich zu einer vollkommen homogenen Lamelle 
auf einander lagern, Auf Durchschnitten der Augen von Schafsembryonen ron $32 \mathrm{~mm}$ Scheitelsteisslänge findet man die Membrana Descemetii schon angelegt, erkemnt aber noch deutlich ihre fibrilläre Struktur und ihren hie und da zweischichtigen Charakter. Die linsenwärts gelegene Zellschichte wird zum D escem et schen Epithel. Man kam aber die erste Anlage der Descemetschen Membran mit Epithel bei Schafsembryonen ron $26 \mathrm{~mm}$ Scheitelsteisslänge nicht nur vor der Linse beobachten, sondern sie lässt sich auch noch oin Stück weit in das scheinbar noch nicht geschiedene Gerrebe von Iris und Cornea rerfolgen. Es geht also der Anlage der Vorderkammer die Anlage der Descemetschen Membran samt ihrem Epithel roraus, das man an Flächenschnitten ganz gut an seinen besonderen Kernformen und der Abgrenzung der einzehnen Zellterritorien erkennt. Bei Embryonen von $32 \mathrm{~mm}$ Scheitelsteisslänge ist, wie in der Anmerkung Seite 14 festgestellt wurde, eine schmale Vorderkammer bereits vorhanden, es ist aber auch die Descemetsche Lamelle mit Epithel bercits angelegt.

Der Zellbelag zeigt zu diescr Zeit noch nicht die regelmässige Anordnung, wie wir sie am erwachsenen Tiere finden. Die Zellen liegen bei Schafsembryonen ron $26 \mathrm{~mm}$ Scheitelsteisslänge, in ungleichmässiger Entfernung, sie sind auch noch nicht aneinander geschlossen. In der Grösse der Kerne sind ziemliche Differenzen vorhanden, ihre Form ist sehr viel gestaltig. Von runden bis zu nierenförmigen Figuren kann man alle C̈bergänge finden. Die regelmässige, eigenthümliche Gestaltung der Kerne, wie sie Ballowitz ${ }^{1}$ ) beschrieben hat, ist also zu dieser Zeit noch nicht rorhanden.

Dagegen zeigten Flächenschnitte von Schafsembryonen ron $62 \mathrm{~mm}$ Scheitelsteisslänge ein ganz regelmässiges Zellmosaik.

1) E. Ballowitz. Über das Epithel der Nembrana elastica posterior des Auges, seine Kerne und eine merkwürdige Struktur seiner grossen Zellsphären; im Archiv 1. mikr. Anat. u. Entwgesch., Bd. 56, 1900. 
Die Kerne waren indes auch hier in ihrer Gestalt noch polymorph. In einigen Zellen dieses Stadiums konnte bereits die Anlage einer Sphäre, wie sie von Ballowitz (l. c.) beschrieben und abgebildet wurden, beobaclitet werden. In der Tafel Fig. 5 sind 2 von den Zellen dieses Stadiums wiedergegeben. In der Zelle a gingen von dem einen Centralkörper zwei sehr scharf konturierte gebogene Linien aus, die am Kernrand versehwanden. Es konnte dieses Bild öfters beobachtet werden. Es ist auffällig, dass hier die Centralkörper zu fädigen Gebilden in Beziehung stehen, während sie in der ausgebildeten Korbsphäre freiliegen. Die Zelle zeigte einen dunkleren Hof, der an den Kern angrenzte. In ihm liegt das Centralkörperpaar. Der Hof selbst zeigt, wenn auch in geringerem Grade, die eigentümlichen Strukturverhältnisse, wie sie schon von Ballowitz eingehend geschildert sind.

Nach diesen Ausführungen über die Descemetsche Membran und ihr Epithel kann ich mich mit den Annahmen von Arnold (1. c. p. 6) nicht einverstanden erklären. Nach ihm soll das Descemetsche Epithel erst nach Bildung der vorderen Augenkammer entstehen. Er sagt: "Der ganze Vorgang macht den Eindruck, als ob das Epithel diesem Trennungsprozess seine Entstehung verdanke, als ob diese wesentlich von dem Eintreten der ersteren abhänge«. Mir scheint das Verhältnis gerade umgekehrt zu sein. Zuerst entwickelt sich Membran mit Epithel und daraufhin erfolgt eine Abspaltung das Irisstromas an der Epithelfläche, wesentlich hervorgerufen durch die in der Anmerkung Seite 14 geschilderten Bedingungen.

\section{Vorderes Cornealepithel.}

Ich wende mich nun zur Beschreibung des vorderen Epithels der Cornea. Bei jungen Stadien von Schafsembryonen von 15 und $22 \mathrm{~mm}$ Scheitelsteisslänge war nur ein einschichtiges Epithel vorhanden. Die Zellen haben ungefähr 
cubische Form. Anders gestalten sich schon die Verhältnisse bei Schafsembryonen von 26,29 und $32 \mathrm{~mm}$ Scheitelsteisslänge. Auf der cubischen Zelllage findet man noch eine äusserst dünne Lamelle von ganz flachen Zellen. Die Länge des einzelnen Elementes beträgt etwa das 3-4fache einer cubischen Zelle. Mit starken Vergrösserungen kann man hier schon an einander zugewandten Seiten gegen die Basis zu protoplasmatische Brücken wahrnehmen. Man findet auch Protoplasmafortsätze von der Basis in das unterliegende Bindegewebe abgehen, wie

Anmerkung. Beiläufig sei hier eine Beobachtung erwähnt, die eigentlich ausserhalb der Grenzen der vorliegenden Arbeit liegt, die sich aber bei der Untersuchung fertiger Hornhäute zufällig nebenbei ergeben hat. Trägt man nämlich durch einen Rundschnitt Cornea und Iris vom fixierten Bulbus ab, so springt, wenn ringsum der Schnitt die Linse mitgetroffen hat, die vordere Linsenkapsel mit mehr oder weniger Epithel ab. So abgezogene Linsenkapseln mit ihrem aufgelagerten Epithel sind schon von Rabl untersucht. Rabl schreibt auf Seite 36, III. Teil der Arbeit über die Linse: ๖Es ist sehr auffallend, dass die Kerne, wenn überhaupt, so nur in den seltensten Fällen genau in der Mitte der Zellareale liegen; weitaus in der Mehrzahl der Fälle haben sie eine excentrische Lage und manchmal liegen sie dicht an der Grenze der betreffenden Areale. Diese Erscheinung ist mir ganz besonders am Linsenepithel des Rindes und Hundes aufgefallen, die sich in vorzüglicher Weise zu solchen Untersuchungen eignen *. Färbt man solche abgesprengte Linsenkapseln mit Eisenalaunhämatoxylin, so ergibt sich der merkwürdige Befund, dass die Centralkörper, die mit grosser Deutlichkeit in jeder Zelle vorhanden sind, ebenfalls sehr oft eine solche Randlage im Bereiche des betreffenden Zellgebietes einnehmen. Sie liegen immer möglichst weit weg vom Kern am entgegengesetzten Randtheil der Zelle. Zwischen Kern- und Centralkörper ist dann eine grosse Strecke freien Protroplasmas. Besonders bemerkenswert ist auch die Lage der Centralkörperpaare zu einander in benachbarten Zellen. Gewöhnlich liegen sie dicht an der Zellgrenze einander direkt gegenüber. Stossen 3 Zellen in einem Punkte zusammen, so werden die Centrosomen sehr oft ganz vorne in den Spitzen der Winkel angetroffen, die durch die sich begrenzenden Zellen gebildet werden. Sie liegen also auch hier in möglichster Nähe zu einander. An solchen Stellen waren auch Verdickungen der Grenzlinien zu sehen. 
die Fig. 3 auf der Tafel von einem Embryo von $32 \mathrm{~mm}$ Scheitelsteisslänge zeigt. Die Kerne der Epithelzellen sind im Verhältnis zum Zellleib gross, gewöhnlich sind mehrere stark gefärbte Nukleolen in denselben vorhanden. Das Protoplasma ist sehr spärlich um die Kerne gelagert und füllt manchmal nur die Kernbuchtungen aus. Etwas grössere Anhäufungen findet man da, wo sich Fibrillen mit den Zellen rerbinden. Bei Schweinsembryonen von 40 und $46 \mathrm{~mm}$ Scheitelsteisslänge war das Epithel noch zweischichtig. Doch lagen die Kerne der cubischen Zellen nicht mehr in gleicher Höhe, sonderu es liess sich eine tiefere und höhere Reihe unterscheiden. Wir haben also hier ein zweischichtiges Epithel und auf diesem die flachen Zellen, wie sie in den rorhergehenden Stadien vom Schaf beschrieben wurden. Protoplasmabrücken waren wegen der pallisadenförmigen Aneinanderlagerung der Zellen nicht zu sehen. Doch erstreckten sich hie und da Ausläufer in das unterliegende Gerrebe. Schafsembryonen ron $62 \mathrm{~mm}$ Scheitelsteisslänge zeigten bereits ein mehrschichtiges Epithel entwickelt. Schon bei schrachen Vergrösserungen war deutlich zu sehen, dass es sich aus 2 verschiedenen Elementen zusammensetzte. Zum grossen Teil waren es Zellen, die in ihrer Form und Tinktion den vorher beschriebenen glichen. Dazwischen aber waren schlanke, dunkel gefärbte Elemente eingeschoben, die mit ihrer Längsachse in den meisten Fällen senkrecht zur Basis standen. Dieselbe Beobachtung hat schon Rabl (l. c. III. Teil) am Linsenepithel bei einem Kaninchen ron $47 \mathrm{~mm}$ Länge gemacht. Sehr schön waren bei dem Schafe ron $62 \mathrm{~mm}$ die protoplasmatischen Terbindungen zwischen den Zellen zu sehen. Auch in das unterliegende Gewebe erstreckten sich zahlreiche Ausläufer.

Das Epithel der fertigen Hornhaut besteht aus einer Reihe von Schichten über einander gelagerter Zellen, die gegen die Oberfläche hin die typischen, schon in der Literatur beschriebenen Terändcrungen zeigen. Bei fast ausgetragenen Hunden 
und Kälbern finden sich zahlreiche Teilungsfiguren, die aber fast alle nicht in der tiefsten Zellschichte, sondern verstreut in den darauf folgenden anzutreffen sind. Ein ganz besonderes Verhalten zeigte die Basalschichte, wovon in der Zeichnung 6 auf der Tafel ein Bild entworfen wurde. Man findet nämlich auf Schrägschnitten, dass sich die basalen Epithelzellen mit ungemein langen Ausläufern in das unterliegende Fibrillengewebe hereinziehen. Es ist mir nicht gelungen, auch mit Anwendung der besten optischen Hülfsmittel und der verschiedensten Fixierungs- und Färbeverfahren nicht, etwas bestimmtes über einen Zusammenhang mit den fibrillären Gebilden ermitteln zu können. Es lässt sich nur soviel sagen, dass diese Fortsätze, immer dünner und zarter werdend, sich allmählich in dem Fibrillengewirr verlieren.

Ich fasse die Resultate der Arbeit kurz zusammen, wie folgt :

1. Als grob mechanische Faktoren in der Genese der Cornea sind mit Sicherhcit anzusprechen intraocularer Druck und Druck der Lider während ihres Wachstums und Geschlossenseins.

2. Das fibrilläre Gewebe der Cornea propria cntstelnt als ein Differenzierungsprodukt der an Ort und Stelle befindlichen Zellen, die später zu den Hormhautzellen merden.

3. Zur regehmässigen Gewebsanordnung tragen wesentlich die in 1 genannten Faktoren bei.

4. Die Torderkammer und die Eigenwölbung der Cornea sind in ihrer Entstehung mit der Bildung des Corpus ciliare rerknüpft.

5. Das Ejithel der Cornea steht zu gewissen Zeiten der Genese in direkter Verbindung mit dem subepithelialen Fibrillengeflecht. 
6. Die Descemetsche Membran entsteht (bei Schafsembryonen von $26 \mathrm{~mm}$ Scheitelsteisslänge) aus 2 Zellschichten, von denen die innere zum Epithel wird.

7. Descemetsche Membran und ihr Epithel sind vor dem Entstehen der vorderen Augenkammer bereits angelegt. Damit ist bereits eine Scheidung des Gewebes in Irisstroma und Cornea vollzogen.

Schliesslich entledige ich mich der angenehmen Pflicht, Herrn Professor L. Gerlach lür die Überlassung der Hilfsmittel des anatomischen Instituts und für das wohlwollende Interesse, das er für meine Arbeiten zeigte, meinen Dank auszusprechen, sowie Herrn Dr. Spuler für die Anregung zu dieser Arbeit, seinen liebenswürdigen Rat bei den histiologischen Untersuchungen, der Deutung der Präparate, und der Abfassung dieser Studien. 


\section{Frrklärung der Abbildungen.}

Die Zeichnungen sind mit Seibert's Apochromat $2 \mathrm{~mm}$ (Apertur 1,30) und Ocular 12 in Objekttischhöhe entworfen, mit Ausnahme von Fig. 5.

1. Bindegewebszelle aus einem Flächenschnitt der Cornea eines Schafsembryos von $26 \mathrm{~mm}$ Scheitelsteisslänge mit Fibrillenbildung. Bei $\mathrm{X}$ ein bis an den Kern herangehender Fibrillenzug. (Färbung nach Heidenhain.)

2. Zelle aus einem Flächenschnitt der Cornea eines Kalbes. Zahlreiche Protoplasmaausläufer, die ringsum mit den Nachbarzellen in Verbindung stehen. (Färbung nach Heidenhain.)

3. Schrägschnitt durch den basalen Teil des äusseren Hornhautepithels eines Schafembryo von $32 \mathrm{~mm}$ Scheitelsteisslänge. Es sind direkte Verbindungen der Fibrillen mit den Epithelzellen zu sehen. (Färbung nach $\mathrm{Heidenhain} \mathrm{mit} \mathrm{nachfolgender} \mathrm{Rubin} \mathrm{S-Behandlung.)}$

4. Linsenkapsel mit Anlage der Descemetschen Membran mit Epithel aus einem Durchschnitt durch das Ange eines Schafsembryos von $29 \mathrm{~mm}$ Scheitelsteisslänge. a) Membrana propria lentis. b) Membrana vasculosa lentis. c) Anlage der Descemetschen Membran mit Epithel. d) An 2 Stellen sind collagene Massen von roter Färbung zu sehen, ein Produkt des Zellprotoplasmas. (Färbung nach Heiden hain mit nachfolgender Rubin S-Behandlung.)

5. Zwei Zellen aus einem Flächenschnitt des Descemetschen Epithels von dem Auge eines Schafsembryos von $62 \mathrm{~mm}$ Scheitelsteisslänge. (Färbung nach $\mathrm{Heidenhain} \mathrm{mit} \mathrm{nachfolgender} \mathrm{Rubin}$ S-Behandlung.) Gez. mit Ölimmersion 1/12 (S e i ber t) und Ocular III.

6. Schrägschnitt aus der Basalschichte des Hornhautepithels eines Kalbes. Protoplasmaausläufer in das subepitheliale Fibrillengewebe. (Färbung nach Heidenhain mit nachfolgender Rubin S-Behandlung.) 